\title{
Prevalência de infecções bucais em ambiente hospitalar
}

\section{Prevalence of oral infections in a hospital environment}

\author{
Lara Maria Moreira de Faria $^{1}$ (D) $\mid$ Christiany Beatriz Cordeiro ${ }^{1}$ (iD $\mid$ Gessiane de Fátima Gomes $^{2}$ \\ Valéria da Silva Baracho $^{2}$ (D) | Elayne Cristina Ferreira de Aguiar $^{2}$ (D) | Evandro Silveira de Oliveira ${ }^{1}$ (D) \\ Dhelfeson Willya Douglas-de-Oliveira ${ }^{1}$ (D) $\mid$ Patricia Furtado Gonçalves ${ }^{1}$ (D) | Olga Dumont Flecha ${ }^{1}$ (iD).
}

\section{G ACESSO LIVRE}

${ }^{1}$ Departamento de Odontologia, Faculdade de Ciências Biológicas e da Saúde,

Universidade Federal dos Vales do Jequitinhonha e Mucuri,

Diamantina, Minas

Gerais, Brasil.

${ }^{2}$ Hospital Santa Casa de Caridade, Diamantina, Minas Gerais, Brasil.

\section{Citação}

Moreira de faria LM, Cordeiro CB, Gomes GF, Silva Baracho V, Ferreria de Aguiar EC, Silvira de Oliveira E, Douglas-de-Oliveira DW, Furtado Gonçalves P, Dumont Flecha O. Prevalência de infecções bucais em ambiente hospitalar. Rev Estomatol. 2020;28(1):8-16. DOI: $10.25100 /$ re.v28i.1.105661

\section{Correspondência}

Lara Maria Moreira de Faria. Rua da Glória, 187 - Centro, Diamantina Minas Gerais, Brasil. CEP: 39100-000 Fone: +55 (38) 3532-6082. E-mail: laramfaria_@hotmail.com

\section{Copyright:}

(C) Universidad del Valle.

\section{RESUMO}

Objetivo: Verificar a prevalência de infecções bucais em ambiente hospitalar no período de 13 meses.

Materiais e métodos: Estudo transversal, realizado no Centro de Terapia Intensiva (CTI) e na Clínica Neurológica da Santa Casa de Caridade de Diamantina, Minas Gerais, Brasil. Foram analisados todos os dados referentes às infecções bucais e hospitalares, ocorridas no período de março de 2017 a março de 2018, obtidos pela Comissão de Controle de Infecção Hospitalar da instituição. A população estudada compreendeu todos os pacientes desses setores, com idade acima de 16 anos, de ambos os sexos e que tiveram infecção bucal após 48 horas de internação.

Resultados: Dentro do total de 2.703 pacientes a prevalência de infecção bucal foi de $1,15 \%$ ( $\mathrm{n}=31)$ dentro do período de 13 meses, sendo a candidíase de maior ocorrência. A prevalência de infecção hospitalar foi de 2,85\% . Conclusão: A prevalência das infecções bucais foi maior no CTI, em comparação à Clínica Neurológica, sendo a infecção mais prevalente a candidíase oral. Sugere-se maior atenção à saúde bucal dos pacientes hospitalizados, diante da maior vulnerabilidade dos mesmos às infecções bucais.

\section{PALAVRAS-CHAVE}

Infecção hospitalar; saúde bucal; equipe hospitalar de odontologia; pacientes internados; unidade hospitalar de odontologia.

\begin{abstract}
Aim: To verify the oral infections prevalence in a hospital environment within a period of 13 months.

Materials and methods: A cross-sectional study was carried out at the Intensive Care Center and Neurological Clinic of Santa Casa de Caridade, in Diamantina, Minas Gerais, Brasil. All data referring to oral and hospital infections occurred in the period from March 2017 to March 2018 were obtained. The research source was the Hospital Infection Control Committee of the institution. The sample comprised all the patients of these sectors, aged over 16 years, of both genders and who had oral infection after 48 hours of hospitalization.

Results: Within 2703 patients, the oral infection prevalence was $1.15 \%(\mathrm{n}=31)$ within 13 months, being candidiasis the most frequent. The prevalence of nosocomial infection was $2.85 \%$.

Conclusion: The prevalence of oral infections was higher in the Intensive Care Center compared to Neurological Clinic, being candidiasis the most common oral infection. More attention should be given to the hospitalized patients's oral health, considering their greater vulnerability to oral infections.
\end{abstract}

\section{KEYWORDS}

Hospital infection; Oral health; hospital dental team, hospitalized patients; hospital dental unit. 


\section{INTRODUÇÃO}

A infecção hospitalar (IH) é definida como aquela adquirida após a admissão do paciente e que se manifesta durante a internação ou mesmo após a alta, quando pode ser relacionada com a hospitalização ou procedimentos hospitalares, sendo as infecções bucais um tipo de IH. Todas as infecções devem ser notificadas após 48 horas da admissão do paciente, ou até 48 horas após a alta do mesmo. ${ }^{1}$

Superfícies mucosas humanas são habitadas por um conjunto diversificado de microrganismos. Estes incluem não apenas bactérias, mas também eucariontes unicelulares, como as leveduras que podem desenvolver infecções fúngicas em ambiente hospitalar, ${ }^{2}$ sendo que essas passaram a ser de grande importância ao longo dos anos, pelo seu aumento progressivo e elevadas taxas de mortalidade e morbidade entre os pacientes. ${ }^{3,4}$

Microrganismos patogênicos no biofilme dentário têm sido implicados em processos infecciosos e / ou inflamatórios, ${ }^{5}$ sendo que úlceras, lesões cariosas e bolsas periodontais configuram focos de infecções bucais. Dessa forma, podem disseminar microrganismos para órgãos e tecidos, principalmente em pacientes imunocomprometidos e estão relacionados ao pior estado de saúde sistêmico em pacientes hospitalizados. ${ }^{6,7,8}$

Estudos têm demonstrado a influência da microbiota bucal nos diversos meios teciduais e apontam forte associação entre esses patógenos, que atuam como foco primário de infecção e quadros infecciosos à distância, provocando consequentemente doenças sistêmicas, tais como complicações cardíacas coronárias, acidentes vasculares encefálicos, endocardite bacteriana, diabetes mellitus e infecção respiratória..$^{9,10,11}$

A ocorrência de infecções hospitalares pode determinar um aumento no tempo e custos de internação e nos índices de mortalidade na população acometida. $^{12}$ Relatos anteriores sugeriram que os profissionais que trabalham em hospitais brasileiros dão pouca credibilidade aos procedimentos odontológicos, como a higiene bucal dos pacientes internados, e geralmente não reconhecem a íntima associação entre higiene bucal e prevenção de doenças sistêmicas. ${ }^{13}$

Já existem regulamentações e projetos de lei que estabelecem a obrigatoriedade de haver cirurgiões dentistas integrados às equipes hospitalares, sendo responsáveis pelo monitoramento da saúde bucal dos pacientes internados; mas apesar de isso não ser realidade em muitos hospitais brasileiros da rede pública e privada, esses projetos tendem a se fortalecer. ${ }^{14}$

Pesquisas são essenciais para que se possa obter dados etiológicos e epidemiológicos sobre infecções bucais no ambiente hospitalar, além de informações sobre seu prognóstico e manejo, a fim de evitar ou reduzir o tempo de internação. A análise de dados permite que sejam planejadas ações de saúde, a avaliação da efetividade dos serviços e para comparar prevalências em locais e períodos diferentes. ${ }^{15,16}$

Poucos são os estudos sobre a frequência de infecções bucais em um ambiente hospitalar. As investigações publicadas anteriormente são apenas sobre infecções odontogênicas que incluem um pequeno número de pacientes internados. ${ }^{17-21}$

Assim, o objetivo principal deste estudo foi verificar a prevalência de infecções bucais, e demais infecções hospitalares em um hospital de médio porte, durante o período de 13 meses, em que havia dentistas residentes na instituição.

Quanto à infecção bucal, foram também descritos os tipos e localização das infecções, o setor e tempo de internação, dados referentes ao sexo, idade, uso de próteses removíveis e tratamentos utilizados.

\section{MATERIAIS E MÉTODOS}

O presente trabalho trata-se de um estudo transversal descritivo em que foram analisados todos os dados referentes às infecções bucais, sendo o objetivo principal dessa pesquisa e dados gerais sobre as infecções hospitalares.

O período do estudo foi de 13 meses, considerando-se o primeiro dia de março de 2017 até o último dia de março de 2018, sendo realizado na Santa Casa de Caridade de Diamantina, Minas Gerais, Brasil, um hospital de médio-porte, que atende Diamantina e outros 31 municípios da região.

Os registros foram obtidos por meio da Comissão de Controle de Infecção Hospitalar (CCIH), responsável pelo monitoramento e notificação das infecções na instituição.

Para a condução do mesmo, um parecer favorável foi emitido pelo Comitê de Ética em Pesquisa da Universidade Federal dos Vales do Jequitinhonha e Mucuri (UFVJM), com o número 2.948.884 e posteriormente firmou-se a carta de anuência com o hospital, autorizando o acesso às informações. 
Todos os princípios éticos da pesquisa envolvendo seres humanos foram seguidos em seu delineamento, de acordo com as leis vigentes no país e a Declaração de Helsinki de 1975, revisada em 2013.

Apesar de a instituição oferecer atendimento à pacientes de todas as faixas etárias, a população amostral incluída na pesquisa foram aqueles com idade superior a 16 anos, de ambos os sexos e que apresentaram manifestação de infecção bucal e demais infecções hospitalares após 48 horas de internação durante o referido período do estudo, não havendo outros motivos de exclusão de pacientes.

Em relação aos dados de infecção hospitalar, foram considerados todos os setores da instituição, bem como a sua prevalência global. Já os dados específicos de infecções bucais direcionaram-se aos pacientes internados nos setores de alta complexidade, sendo a Clínica Neurológica e o Centro de Terapia Intensiva (CTI), uma vez que ambos contavam com a avaliação diária de dois cirurgiões dentistas do programa de residência que atende a instituição, os quais além de avaliarem, também realizavam a higiene bucal dos pacientes, o diagnóstico e tratamento das infecções bucais encontradas.

Os residentes já apresentavam alguns anos de formação, obtendo experiência clínica suficiente na área odontológica e eram diretamente supervisionados por professores especialistas da UFVJM, como estomatologistas e periodontistas.

Para o diagnóstico das infecções bucais, os pacientes internados passavam por um exame clínico odontológico intrabucal, com avaliação das superfícies mucosas e dentais, que era realizado diariamente pelos dentistas residentes, integrados às equipes multiprofissionais.

O exame era exclusivamente clínico e realizado por meio do uso de espátulas de madeira desde o primeiro dia da admissão dos pacientes nos dois setores, e a partir disso, diante dos sinais e sintomas de toda e qualquer manifestação de infecção bucal, diagnosticada após dois dias completos da admissão dos mesmos, era então feita a notificação à CCIH.

Quanto aos cuidados de higiene bucal, seguiu-se o protocolo da Sociedade Brasileira de Pneumologia e Tisiologia (SBPT), a qual preconiza para pacientes intubar a administração de digluconato de clorexidina a $0,12 \%$, de 12 em 12 horas e para os não entubados, a escovação dental de 8 em 8 horas, sendo importante ressaltar que a maioria dos pacientes internados nesses setores já havia passado por algum período de intubação.

Em relação aos pacientes acometidos, além da prevalência de infecções bucais, foram também coletadas informações sobre a idade, sexo, setor de internação, tempo de internação, local de ocorrência da infecção, tipo de infecção, dados sobre o uso de próteses removíveis e a conduta adotada.

De acordo com desenho do estudo, houve dispensa do Termo de Consentimento Livre e Esclarecido (TCLE), visto que, em nenhum momento os pesquisadores tiveram acesso a nome e dados pessoais dos pacientes, pois foram analisados apenas índices gerados pelo sistema de notificação da instituição.

Os dados coletados foram analisados pelo software SPSS $^{\circledR}$ (Statistical Package for Social Sciences, IBM Inc., USA) versão 25. Foram realizadas análises de estatística descritiva para obtenção de frequência, média e desvio-padrão. A normalidade dos dados foi verificada pelo teste de ShapiroWilk. A associação entre as variáveis categóricas e quantitativas foram feitas por meio do teste QuiQuadrado e teste $\mathrm{T}$ de student, respectivamente. Foi adotado o nível de significância correspondente à $\mathrm{p}<0,05$.

A taxa de prevalência foi definida como a razão de pessoas acometidas por infecção pelo total de internados.

\section{RESULTADOS}

Em um total de 2.703 pacientes internados no CTI e na Clínica Neurológica, 31 desses apresentaram infecção bucal, sendo a prevalência de $1,15 \%$ $(\mathrm{n}=31)$ dentro do período de 13 meses (Tabela 1).

Considerando os 31 pacientes que tiveram infecção bucal, a média de idade calculada foi de 68,55 $( \pm 18,63)$ anos e o tempo médio de internação foi de $23,13( \pm 13,62)$ dias.

A prevalência das infecções bucais foi maior no sexo feminino, com um total de $21(67,70 \%)$ mulheres e quanto ao setor, o maior número das notificações foi no CTI, havendo $18(58,10 \%)$ pacientes com infecção bucal, seguido de uma menor prevalência na Clínica Neurológica, com $41,90 \%$ dos casos (Tabela 2).

A candidíase foi a infecção bucal mais prevalente, sendo que dentro dos 31 pacientes essa lesão foi diagnosticada em $25(80,60 \%)$ dos mesmos, 
seguida pela queilite angular que estava presente em $3(9,70 \%)$ pacientes.

O local mais comum da manifestação das lesões foi na região de língua, em 18 (34,60\%) pacientes, seguida da região de mucosa jugal em $12(23,10 \%)$ pacientes. $\mathrm{O}$ tratamento mais utilizado para manejo das infecções foi o medicamentoso, administrado em $28(90,30 \%)$ casos (Tabela 2).

Em relação aos 25 pacientes diagnosticados com candidíase oral, observou-se a maior ocorrência da mesma entre o sexo feminino, acometendo 17 $(68,00 \%)$ mulheres e quanto ao uso de prótese removível, foi verificado que $12(48,00 \%)$ pacientes não utilizavam a mesma quando diagnosticados com a infecção.
Não existiram associações significativas entre os dados de candidíase e: sexo $(\mathrm{p}=0,999)$, uso de prótese $(\mathrm{p}=0,673)$ e tempo de internação $(\mathrm{p}=0,095)$. Houve associação estatisticamente significante entre candidíase e idade $(\mathrm{p}<0,001)$ (Tabela 3).

Quanto à média de idade dos pacientes com candidíase oral, a mesma foi de 74,36 $( \pm 13,99)$ anos, e em relação ao tempo de internação, pacientes com candidíase apresentaram uma média de 21,12 ( $\pm 12,33)$ dias, e pacientes sem candidíase apresentaram um tempo maior de internação, com a média de 31,50 $( \pm 16,68)$ dias (Tabela 3$)$.

Tabela 1. Distribuição dos pacientes com infecção bucal.

\begin{tabular}{lll}
\hline Setor & \multicolumn{2}{l}{ Prevalência } \\
\hline & \multicolumn{1}{c}{$\mathbf{n}$} \\
\cline { 2 - 2 } CTI & 18 & 1,52 \\
Clínica Neurológica & 13 & 0,85 \\
CTI e Clínica Neurológica & 31 & 1,15 \\
& & \\
Total de Pacientes & & \\
CTI & 1180 \\
Clínica Neurológica & 1523 \\
CTI e Clínica Neurológica & 2703 \\
\hline
\end{tabular}

Tabela 2. Características dos pacientes com infecção bucal.

\begin{tabular}{lcc}
\hline Variável & Média & Dp \\
\hline Idade (anos) & 68,55 & 18,63 \\
Tempo de internação (dias) & 23,13 & 13,62
\end{tabular}

\section{Sexo}

Masculino

Feminino n

10

21
$\%$

32,30

67,70

\section{Setor}

CTI

Clínica Neurológica

\section{Tratamento}


Moreira de Faria e cols.

Laser (Herpes simples e mucosite)

$03 \quad 9,70$

Medicamentoso

$28 \quad 90,30$

Nistatina e Fluconazol (Candidíase pseudomembranosa)

$25 \quad 89,30$

Omcilon-AM (Queilite Angular)

$03 \quad 10,70$

\begin{tabular}{lll} 
Classificação da lesão & 01 & 3,20 \\
Mucosite & 25 & 80,60 \\
Candidíase pseudomembranosa & 03 & 9,70 \\
Queilite Angular & 02 & 6,50 \\
Herpes simples & & \\
& & \\
Localização das lesões & 9,60 \\
Palato & 05 & 3,80 \\
Arco glossofaríngeo & 02 & 34,60 \\
Língua & 18 & 9,60 \\
Assoalho bucal & 05 \\
Mucosa jugal & 12 & 23,10 \\
Rebordo alveolar & 7,70 \\
Comissura labial & 04 & 9,60 \\
Lábio & 05 & 1,90 \\
\hline
\end{tabular}

Tabela 3. Associação da candidíase com variáveis independentes.

$\begin{array}{lll}\text { Com candidíase } & \text { Sem candidíase }\end{array}$

$\begin{array}{lccc}\text { Sexo } & \mathbf{n}(\%) & \mathbf{n}(\%) & 0,999 * \\ \text { Masculino } & 8(32,00) & 2(33,30) & 4(66,70) \\ \text { Feminino } & 17(68,00) & \\ \text { Uso de Prótese } & 10(40,00) & 2(33,30) & 0,673^{*} \\ \text { Sim } & 12(48,00) & 4(66,70) & 0(0,00)\end{array}$

Média (DP)

Média (DP) 


\begin{tabular}{lccc} 
Idade (anos) & $74,36(13,99)$ & $44,33(16,44)$ & $<\mathbf{0 , 0 0 1 * *}$ \\
Tempo de internação (dias) & $21,12(12,33)$ & $31,50(16,68)$ & $0,095^{* *}$ \\
\hline$*$ Qui-Quadrado **Teste t & &
\end{tabular}

Observou-se que em um total de 5.747 pacientes internados na Santa Casa no período de 13 meses, 164 foram notificados com IH sendo a prevalência de $2,85 \%$, considerando-se todos os setores da instituição. A prevalência das IH no CTI foi de $8,05 \%$, considerando os 1.180 pacientes que passaram por esse setor no período do estudo, sendo esse o setor de maior prevalência (Tabela 4).

Tabela 4. Distribuição dos pacientes com infecção hospitalar.

\begin{tabular}{|c|c|c|}
\hline \multirow[t]{2}{*}{ Setor } & \multicolumn{2}{|c|}{ Prevalência } \\
\hline & $\mathbf{n}$ & $\%$ \\
\hline CTI & 95 & 8,05 \\
\hline Clínica Neurológica & 14 & 0,91 \\
\hline Outros Setores & 55 & 1,83 \\
\hline Geral na Instituição & 164 & 2,85 \\
\hline \multicolumn{3}{|l|}{ Total de Pacientes } \\
\hline CTI & 1180 & \\
\hline Clínica Neurológica & 1523 & \\
\hline Outros Setores & 3044 & \\
\hline Geral na Instituição & 5747 & \\
\hline
\end{tabular}

Nenhum paciente da Clínica Neurológica e CTI teve as duas infecções (bucal e hospitalar) simultaneamente.

\section{DISCUSSÃO}

Constatou-se que a prevalência de infecções bucais em ambiente hospitalar foi de 1,15\% $(n=31)$, considerando-se todos os pacientes que foram internados no período em questão nas duas clínicas, sendo a maior ocorrência no CTI. Diante das reduzidas evidências relatadas na literatura, não foram encontrados dados seguros que permitissem uma comparação com os resultados aqui apresentados.

A literatura mostra que pacientes hospitalizados apresentam maior prevalência de candidíase bucal em razão de alterações sistêmicas que podem modificar o microambiente oral, favorecendo a ocorrência de infecções oportunistas como a candidíase, ${ }^{22,23}$ sendo uma das infecções fúngicas que mais acomete os pacientes em unidades hospitalares. ${ }^{24}$ Isso é verificado pelos resultados aqui apresentados que demonstram sua ocorrência em 80,60\% dos casos de infecção.
Em estudos realizados por Grimoud et al. ${ }^{22}$ em um Centro Hospitalar da Universidade de Toulouse, a candidíase oral também foi a infecção bucal de maior prevalência nos pacientes ali internados, ocorrendo em 67,30\% dos mesmos. Em contrapartida, estudos realizados em dois hospitais de Curitiba, indicaram uma porcentagem inferior da candidíase oral, sendo a mesma presente em apenas $30 \%$ dos pacientes internados. $^{25}$

De acordo com estudos de Meira et al. ${ }^{26}$ homens e mulheres foram igualmente afetados pela candidíase oral, dados que se assemelham aos resultados aqui encontrados. Entretanto, Araújo et $a l .{ }^{27}$ afirmam em seus estudos que essa infecção acometeu 3,5 vezes mais mulheres que homens.

Foi visto que entre os 25 pacientes diagnosticados com a candidíase oral, a maioria $(54,50 \%)$ não fazia uso de nenhum tipo de prótese removível, apesar disso, sabe-se que um dos fatores predisponentes para o surgimento da candidíase oral pode ser o uso de próteses removíveis mal adaptadas e mal higienizadas. ${ }^{28}$

Não foi observada nenhuma associação entre a candidíase e o sexo, isso talvez possa se justificar 
devido ao menor número de pacientes considerados no presente trabalho em comparação com os estudos de Meira et al. ${ }^{26}$ e Araújo et al. ${ }^{27}$. Também não foi encontrada associação entre a candidíase e o uso de prótese e candidíase com o tempo de internação.

A prevalência das infecções bucais do presente estudo, foi superior no CTI podendo esse fato ser justificado nesse setor pela condição dos pacientes que geralmente encontram-se com baixa capacidade de realizar uma boa higiene bucal. ${ }^{29}$

Esses pacientes se encontram mais suscetíveis a infecções bucais como a candidíase, onde a aderência fúngica pode estar facilitada pelo baixo $\mathrm{pH}$, higienização deficiente, baixo fluxo salivar e interações com a microbiota, outras explicações podem ser a constante reexposição e reinfecção por leveduras no ambiente hospitalar, a influência de terapia medicamentosa ou ainda a deficiência imunológica. $^{30}$

Na Clínica Neurológica, a prevalência das infecções bucais foi observada em $0,85 \% \quad(n=13)$ dos pacientes. Alguns estudos afirmam que condições neurológicas, como o acidente vascular cerebral, levam a fraqueza física, falta de coordenação e problemas cognitivos, tornando-se difícil manter uma boa higiene bucal ${ }^{31}$ e tal comprometimento aumenta a prevalência de doenças bucais ${ }^{32}$ em pacientes nessas condições.

A média de idade em que se manifestaram as infecções bucais foi de 68,55 anos e a presença da candidíase foi associada à idade. Este resultado é corroborado pelas descobertas de Oliveira et al. ${ }^{33}$ e Fidel et $a l .{ }^{34}$ que afirmam em seus estudos que em pessoas com 60 anos ou mais de idade, o desequilíbrio da microbiota bucal é mais intenso e dessa forma a internação em unidade hospitalar expõe o organismo a condições de vulnerabilidade.

Quanto ao tratamento, os resultados indicaram que houve manejo de 90,30\% $\quad(n=28)$ das infecções bucais com terapia medicamentosa, o que pode estar relacionado à prevalência da candidíase como principal infecção.

No protocolo do presente estudo, os casos de candidíase foram tratados com a Nistatina de 100.000 UI/ML, e se caso não houvesse a remissão total dos sintomas, era então empregado o uso do Fluconazol em dose única, o que está de acordo com as diretrizes da Infectious Diseases Society of America (IDSA), em que há recomendação do fluconazol sistêmico como terapia de primeira linha para o tratamento da candidíase orofaríngea moderada-severa ${ }^{35}$. Nas lesões como herpes simples e mucosite, a laserterapia foi utilizada como manejo, e a queilite angular tratada de maneira tópica com a pomada de Omcilon-AM.

Pacientes críticos internados em terapia intensiva são mais vulneráveis às $\mathrm{IH}$ em comparação com as demais unidades e têm de 5 a 10 vezes mais probabilidade de contraí-la, ${ }^{36}$ o que confirma os resultados aqui apresentados, em que o setor de maior prevalência dessas infecções foi o CTI.

Dentre o total de pacientes internados no CTI, foi visto que $8,05 \%$ dos mesmos apresentaram $\mathrm{IH}$, Isso está de acordo com um estudo realizado no período de 10 meses em uma UTI do Hospital Pró-Clínicas, sendo que, dentre 237 pacientes, 26 tiveram diagnóstico de $\mathrm{IH}$, representando uma prevalência de $10,97 \%,{ }^{37}$ semelhante ao presente trabalho.

Porém essa porcentagem pode variar, como visto em um hospital público do interior do Paraná Brasil, durante um período de 11 meses, que constatou uma maior prevalência de $\mathrm{IH}$ em terapia intensiva, acometendo $27,35 \%$ dos pacientes. ${ }^{38}$

Quanto à prevalência de IH encontrada em todos os pacientes internados no período de 13 meses, a mesma foi de $2,85 \% \quad(n=164)$, enquanto um estudo realizado em um hospital público universitário de Fortaleza, em 2008, apresentou no período de 11 meses uma prevalência média de $8,40 \%$ e o setor de maior ocorrência das IH foi a Clínica Médica I. ${ }^{39}$

A prevalência de infecções bucais encontrada pode ser justificada por alguns fatores como a presença de cirurgiões dentistas que diariamente acompanhavam e higienizavam a cavidade bucal dos pacientes internados em ambos os setores e a existência de um projeto de extensão com ações diárias voltadas à prevenção e promoção de saúde bucal na instituição.

Também é necessário considerar que o diagnóstico das infecções era feito somente por meio dos sinais e sintomas clínicos observados ao exame intrabucal. Sendo assim, existe a possibilidade de terem ocorrido subnotificações, visto que existem infecções com manifestações subclínicas que exigem exames complementares para um adequado diagnóstico e notificação.

O número de setores em que foram avaliadas as infecções bucais pode ser outro fator limitante assim como o tempo de execução do estudo. O ideal seria que todos os setores da instituição 
pudessem ser avaliados, para que houvesse uma adequada comparação entre infecção hospitalar e bucal dentro dos mesmos.

Entretanto foram avaliados os casos de infecção bucal somente no CTI e na Clínica Neurológica, pois ambos contavam com a assistência diária de dentistas, o que não ocorria frequentemente nos demais setores. Além disso, seria ideal acompanhar os setores por um período de tempo maior.

Frente à escassez de estudos nessa área, o presente trabalho contribui com o acréscimo de informações relevantes e enriquecedoras à literatura, abordando comparações pouco discutidas, que servirão como subsídio e incentivo para futuras pesquisas.

\section{CONCLUSÃO}

Pôde-se observar, pelo presente estudo, que infecções bucais oportunistas tendem a se manifestar em pacientes internados, principalmente aqueles em CTI, sendo a candidíase a infecção mais comum, com maior manifestação em região de língua e cuja principal forma de tratamento foi a terapia medicamentosa.

Observa-se a importância da presença de um cirurgião dentista integrado às equipes em ambiente hospitalar possibilitando o reconhecimento de fatores de risco que tornam pacientes internados mais susceptíveis às infecções bucais, bem como a existência de um sistema de notificação das mesmas. Programas de prevenção e promoção de saúde bucal também podem colaborar significativamente para um melhor prognóstico desses pacientes, visto que a saúde bucal não se desvincula da saúde sistêmica.

\section{RELEVÂNCIA CLÍNICA}

As infecções bucais encontradas devem ser consideradas na prevenção destes processos infecciosos e futuras complicações sistêmicas, trazendo benefícios para o paciente e a instituição, através da redução da taxa de mortalidade e dos custos hospitalares. Destaca-se também, a importância do cirurgião dentista dentro do ambiente hospitalar, promovendo interação profissional e melhor qualidade de vida ao paciente.

\section{CONFLITO DE INTERESSES}

Os autores do presente manuscrito declaram a ausência de quaisquer conflitos de interesses.

\section{FONTE DE FINANCIAMENTO}

Financiamento próprio.

\section{REFERÊNCIAS}

1. Garner JS, Jarvis WR, Emori TG, Horan TC, Hughes JM. CDC definitions for nosocomial infections. Am J Infect Control 1988 Jun; 16(3): 128-140.

2. Underhill DM, Iliev ID. The mycobiota: interactions between commensal fungi and the host immune system. Nat Rev Immunol 2014; 14: 405-416.

3. Colombo AL. Epidemiology and treatment of hematogenous candidiasis: a Brazilian perspective. Braz J Infect Dis 2000; 4: 113-118.

4. Pfaller MA. Nosocomial candidiasis: emerging species, reservoirs, and modes of transmission. Clin Infect Dis 1996; 22 (Suppl 2): 89-94.

5. Seneviratne CJ, Zhang CF, Samaranayake LP. Dental plaque biofilm in oral health and disease. Chin J Dent Res 2011; 14(2): 87-94.

6. De Marchi RJ, Hugo FN, Hilgert JB, Padilha DM Association between oral health status and nutritional status in south Brazilian independent-living older people. Nutrition 2008; 24: 546-553.

7. Shimazaki Y, Soh I, Saito T, Yamashita Y, Koga T, Miyazaki $\mathrm{H}$, et al. Influence of dentition status on physical disability, metal impairment, and mortality in institutionalized elderly people. J Dent Res 2001 Jan; 80(1): 340-345.

8. Miguez-Burbano MJ, Ashkin D, Rodriguez A, Duncan $\mathrm{R}$, Flores M, Acosta B, et al. Cellular immune response to pulmonary infections in HIV-infected insividuals hospitalized with diverse grades of immunosuppression. Epidemiol Infect 2006 Apr; 134(2): 271-278.

9. Mealey BL. Influence of periodontal infections on systemic health. Periodontol 2000 1999; 21:197-209.

10. Meyer DH, Fives-Taylor PM. Oral pathogens: From dental plaque to cardiac disease. Curr Opin Microbiolol 1998; 1(1): 88-95.

11. Li X, Kolltveit KM, Tronstad L, Olsen, I. Systemic disease caused by oral infection. Clin Microbiol Rev 2000; 13(4): 547-558.

12. Haley RW. Cost-benefit analysis of infection control programs. In: Bennett JV, Brachman PS. Hospital infection. $4^{\text {th }}$ ed. New York: Lippicont-Raven Publishers; 1998. p. 249-267.

13. Melo Neto JP, Melo MS, dos Santos-Pereira SA Martinez EF, Okajima LS, Saba- Chujfi E. Periodontal infections and community-acquired pneumonia: A case- control study. Eur J Clin Microbiol Infect Dis 2013; 32: 27-32

14. Sousa LVS, Pereira AFV, Silva NBS. A atuação do cirurgião-dentista no atendimento hospitalar. Rev Ciênc Saúde 2014 Jan-jun; 16(1): 39-45.

15. Castellanos PL. A epidemiologia e a organização dos sistemas de saúde. In: Rouquayrol MZ Epidemiologia e saúde. $4^{\mathrm{a}}$ ed. Rio de Janeiro: Medsi; 1993. p.77-84

16. World Health Organization. Oral health surveys: basic methods. $3^{\text {rd }}$ ed. Geneva: Orh/Epid; 1987.

17. Sato FRL, Hajala FAC, Filho FWVF, Moreira RWF, de Moraes M. Eight- Year Retrospective Study of Odontogenic Origin Infections in a Postgraduation 
Program on Oral and Maxillofacial Surgery. J Oral Maxillofac Surg 2009; 67(5): 1092-1097.

18. Dodson TB, Perrott DH, Kaban LB. Pediatric maxillofacial infections: A retrospective study of 113 patients. J Oral Maxillofac Surg 1989 Apr; 47(4): 327 330 .

19. Scutari P Jr, Dodson TB. Epidemiologic review of pediatric and adult maxillofacial infections in hospitalized patients. Oral Surg Oral Med Oral Pathol Oral Radiol Endod 1996; 81: 270-274.

20. Seppänen L, Lauhio A, Lindqvist C, Suuronen R, Rautemaa R. Analysis of systemic and local odontogenic infection complications requiring hospital care. J infects 2008; 57(2): 116-122.

21. Wang J, Ahani A, Pogrel MA. A five-year retrospective study of odontogenic maxillofacial infections in a large urban public hospital. Int $\mathrm{J}$ Oral Maxillofac Surg 2005; 34: 646-649.

22. Grimoud AM, Marty N, Bocquet H, Andrieu S, Lodter JP, Chabanon G. Colonization of the oral cavity by Candida species: risk factors in long-term geriatric care. J Oral Science 2003 Mar; 45(1): 51-55.

23. Meurman JH, Hämäläinen $P$. Oral health and morbidity: implication of oral infections on the elderly. Gerodontology 2006; 23: 3-16.

24. Badiee P, Alborzi A, Joukar M. Molecular assay to detect nosocomial fungal infections in intensive care units. Eur $\mathrm{J}$ Intern Med. European Federation of Internal Medicine 2011; 22(6): 611-615.

25. Stramandinoli RT, Souza PHC, Westphalen FH, Bisinelli JC, Ignácio SA, Yurgel LS. Prevalence of oral candidiasis in hospitalized patients and evaluation of risk factors. Rev Sul-Bras Odontol 2010 Mar; 7(1): 66-72.

26. Meira HC, Oliveira BM, Pereira IF, Naves MD, Mesquita RA, Santos VR. Oral candidiasis: A retrospective study of 276 Brazilian patients. J Oral Maxillofac Pathol 2017 Sep-Dec; 21(3): 351-355.

27. Araújo RR, Rezende AP, Araújo MB, Capistrano HM. Oral candidiasis profile in a clinic of stomatology. Arqu Bras Odontol 2006; 2: 26-31.

28. Myzuka BC. Oral fungal infections. Dent Clin North Am 2005 Jan; 49(1): 49-65.

29. Paju S, Scannapieco FA. Oral biofilms, periodontitis, and pulmonary infections. Oral Dis 2007; 13(6): 508-512.

30. Pires JR, Matareli S, Ferreira RG, Toledo BEC, Zuza EP. Espécies de cândida e a condição bucal de pacientes internados em Unidade de Terapia Intensiva. Rev Assoc Paul Cir Dent 2011; 65(5): 332-337.

31. Arai K, Sumi Y, Uematsu H, Miura $H$ Association between dental health behaviours, mental/physical function and self-feeding ability among the elderly: a cross-sectional survey. Gerodontology 2003; 20: 78-83.

32. Ship JA, Chavez EM. Managerment of systemic diseases and chronic impairments in older adults: oral health considerations. Gen Dent 2000 Sep-Oct; 555-565. 33. Oliveira LCBS, Carneiro PPM, Fischer RG, Tinoco EMB. A presença de patógenos respiratórios no biofilme bucal de pacientes com pneumonia nosocomial. Rev Bras Ter Intensiva 2007; 19(4): 428-433.

34. Fidel Junior RAS, Lourenço RA, Fischer RG. A doença periodontal e o idoso frágil. Revista do Hospital Universitário Pedro Ernesto 2013; 12(1): 1-14.

35. Pappas PG, Kauffman CA, Andes D, Benjamin DK $\mathrm{Jr}$, Calandra TF, Edwards JE Jr, et al. Clinical practice guidelines for the management of candidiasis: 2009 update by the Infectious Diseases Society of America. Clin Infect Dis 2009; 48: 503-535.

36. Vincent JL. Nosocomial infections in adult intensive-care units. Lancet 2003; 361: 2068- 2077.

37. Padrão MC, Monteiro ML, Maciel NR, Viana FFCF, Freitas NA. Prevalência de infecções hospitalares em unidade de terapia intensiva. Rev Bras Clin Med 2010; 8(2): $125-8$.

38. Santos RP, Mariano RL, Takahashi LS, Erdmann MF. Prevalência de Infecção Hospitalar em Unidade de Terapia Intensiva-Um estudo retrospectivo. Rev Enferm UFSM 2014 Abr-Jun; 4(2): 410-418.

39. Nogueira PSF, Moura ERF, Costa MMF, Monteiro WMS, Brondi L. Perfil da Infecção Hospitalar em um Hospital Universitário. Rev enferm UERJ 2009 Jan-Mar; 17(1): 96-101. 